Infusionstherapie 1973-74;1:6

\title{
Impressum
}

Verlag S. Karger Verlag fur Medizin und Naturwissenschaften GmbH, 8340 Germering,

\section{Postfach 2.}

D-8130 Starnberg

Für den wissenschaftlichen Inhalt verantwortliche Herausgeber

Prof. Dr. H. Reissigl, Hofrat

Vorstand des Zentralinstituts für Bluttransfusion und Immuno-

logische Abteilung der Universitätskliniken Innsbruck, an der

Chirurgischen Universitätsklinik, Anichstrasse 35, A-6020,

Innsbruck

Prof. Dr. H. Grobecker

Zentrum für Pharmakologie, Abt. Ill, Johann-Wolfgang-Goethe-Universität, Theodor-Stern-Kai

7, D-6000 Frankfurt/ Main 70

Prof. Dr. U. Henneberg

Geschäftsführender Direktor des Instituts für Anaesthesiologie im Klinikum Steglitz der Freien

Universität Berlin, Hinden-burgdamm 30, D-1 Berlin 45

Prof. Dr. H. G. Lasch

Direktor der Medizinischen Kliniken und Polikliniken der Justus

Liebig-Universität, Klinikstrasse 32b, D-63 Giessen

Wissenschaftlicher Beirat

E.H.Abel, Stade Verwaltung

Dr. W. Bayerl, Bonn Chirurgie

Prim. Prof. Dr. H. Bergmann, Linz Anaesthesie

Dr. H. Boll, Hamm Anaesthesie

Prof. Dr. C. Burri, Ulm Chirurgie

Prof. Dr. H. Busch, Hamburg Bluttransfusion

Prof. Dr. W. Dick, Ulm Anaesthesie

Prof. Dr. R. Dohrmann, Berlin Chirurgie

Prof. Dr. H. Egli, Bonn Bluttransfusion

Prof. Dr. M. Eggstein, Tubingen Innere Medizin

Prof. Dr. C. C. Ehrich, New York Bluttransfusion

Prof. Dr. H.Förster, Frankfurt Biochemie

Prof. Dr. V. Friedberg, MainzGynäkologie

PD Dr. H. E. Franz, Ulm Nephrologie

Dr. P. Frank, Würzburg Pharmazie

Prof. Dr. M. Gemperle, Genf Anaesthesie

Prof. Dr. O. Heidenreich, Aachen Pharmakologie

Prof. A. Heidland, Würzburg Innere Medizin

Dr. P.U.Heuckenkamp, München Innere Medizin

Prof. Dr. G. Hossli, Zurich Anaesthesie 
Prof. Dr. B. Hölscher, Berlin Chirurgie

Prof. Dr. K. F. Kopp, München Nephrologie

Prof. Dr. F. Krück, Homburg/Saar Innere Medizin

Dr. H. J. Kümmell, Melsungen Pharmazie

PD Dr. V. O. Lang, München Pädiatrie

Prof. Dr. A. Lindner, Wien Pharmakologie

Prof. Dr. A. Linke, Ludwigshafen Innere Medizin

Prof. Dr. H. Lutz, Mannheim Anaesthesie

PD Dr. J. Papenberg, Heidelberg Innere .Medizin

Prof. Dr. H.W. Pia, Giessen Neurochirurgie

PD Dr. K. Peter, Mannheim . Anaesthesie

Prof. Dr. G.Rodewald, Hamburg Chirurgie

Prof. Dr. L.Róka, Giessen.- Biochemie

Prof. Dr. E. Rossi, Bern Pädiatrie

Prof. Dr. G. Schettler, Heidelberg Innere Medizin

Dr.H.Schmidt, Frankfurt an... Anaesthesie

Prof. Dr. I. Staib, Darmstadt Chirurgie

PD Dr. H. Stoeckel, Heidelberg ' Anaesthesie

Prof. Dr. W. Vahlensieck, Bonn Urologie

Prof. Dr. K. Wiemers, Freiburg Anaesthesie

Prof. Dr. A. Wretlind, Stockholm Innere Medizin

Prof. Dr. M. Ziegler, Heidelberg Urologie

Prof. Dr. W. E. Zimmermann, Freiburg Chirurgie

Prof. Dr. R. X. Zittel, Ludwigshafen Chirurgie

Schriftleitung

Hans-Dieter Viering

S. Karger Gmbh, Waldschmidstrasse 8 , am See, Tel.: 08151-6866*1/8 $1 / 8$,

Chef vom Dienst

Wolfgang Fischer

Satz: Fotosatz Tutte, Salzweg-Passau. Druck: Ludwig Auer, Donauwörth.

Erscheinungsweise

»Die Infusionstherapie« erscheint zweimonatlich; pro Jahr er-scheint 1 Band zu je 6 Heften; jedes Heft hat ungefähr 90 Seiten Inhalt.

Abonnementspreis

1 Band zu je 6 Heften kostet DM 69,-/US \$24,00/SFr. 72,-. 1 Einzelheft kostet DM 14,-/US

$\$ 4,50 /$ SFr. 14,40, einschliess-lich Mwst., zuzüglich Postgebühren.

Der Abonnementspreis ist im voraus zahlbar. Die Lieferung«der Zeitschrift läuft weiter, wenn sie nicht spätestens 4 Wochen vor Abschluss eines Bandes abbestellt wird.

Sonderhefte sind nur in beschränkter Auflage vorrätig. Der Preis eines Sonderhefts ist abhängig vom Umfang. Abbonnenten er-halten die Sonderhefte zu einem um 20\% vom Ladenpreis ermässigten Vorzugspreis.

Anzeigen

Med.-Pharm. Anzeigenverwaltungs-GmbH\&Co.KG, Waldschmidstrasse 8, D-8130 Starnberg, Tel.: 08151-6866 u. 13299. 


\author{
Abonnemente \\ Abonnementsbestellungen können bei jeder Buchhandlung, bei der Post oder direkt beim Verlag \\ aufgegeben werden: \\ Bundesrepublik DeutschlandiÖsterreich: S. Karger GmbH, Postfach 2, D-8034 \\ Germering/München, Tel.: 0811-844021, Telex: 524865 D, Postscheckkonto: München 40080- \\ 807. \\ Schweiz: S.Karger AG, Arnold-Böcklin-Strasse 25, CH-4011 Basel, Tel.: 061-390880, Telex \\ $62652 \mathrm{CH}$ \\ Frankreich: S. Karger S.A., 42bis, Boulevard de la Tour-Mau-bourg, F-75007 Paris \\ England: John Wiley and Sons Ltd., Baffins Lane, Chichester, \\ USA: Albert J.Phiebig, Inc., P.O. Box 352, White Plains, New York, NY 10602 \\ Australien: Australasian Drug Information Services Pty. Ltd., Agents in Australasia for S. \\ Karger, P.O. Box 194, Balgowlah/ Sydney, NSW 2093
}

